\title{
Dynamic Mapping of Along-Track Ocean Altimetry: Performance from Real Observations 9
}

\author{
MAXime Ballarotta, ${ }^{\mathrm{a}}$ Clément Ubelmann, ${ }^{\mathrm{a}}$ MARINe Rogé, ${ }^{\mathrm{b}}$ Florent Fournier, ${ }^{\mathrm{a}}$ \\ YANnice FAugère, ${ }^{a}$ GÉrAld Dibarboure, ${ }^{c}$ Rosemary Morrow, ${ }^{\mathrm{d}}$ AND NiCOLAT Picot ${ }^{\mathrm{c}}$ \\ ${ }^{\text {a } C o l l e c t e ~ L o c a l i s a t i o n ~ S a t e l l i t e, ~ R a m o n v i l l e ~ S a i n t-A g n e, ~ F r a n c e ~}$ \\ ${ }^{\mathrm{b}}$ Climate Change Research Centre, University of New South Wales, Sydney, New South Wales, Australia \\ ${ }^{\mathrm{c}}$ Centre National d'Etudes Spatiales, Toulouse, France \\ ${ }^{\mathrm{d}}$ LEGOS, Toulouse, France
}

(Manuscript received 3 March 2020, in final form 1 June 2020)

\begin{abstract}
The dynamic optimal interpolation (DOI) method merges altimetric sea surface height (SSH) data into maps that are continuous in time and space. Unlike the traditional linear optimal interpolation (LOI) method, DOI has the advantage of considering a nonlinear temporal propagation of the SSH field. DOI has been successfully applied to along-track pseudo-observations in observing system simulation experiments (OSSEs), demonstrating a reduction in interpolation error in highly turbulent regions compared to LOI mapping. In the present study, we further extend the validation of the DOI method by an observing system experiment (OSE). We applied and validated the DOI approach with real nadir-altimetric observations in four regional configurations. Overall, the qualitative and quantitative assessments of these realistic SSH maps confirm the higher level of performance of the DOI approach in turbulent regions. It is more of a challenge to outperform the conventional LOI mapping in coastal and low-energy regions. Validations against LOI maps distributed by the Copernicus Marine Environment Monitoring Service indicate a 10\%-15\% increase in average performance and an improved resolution limit toward shorter wavelengths. The DOI method also shows improved mesoscale mapping of intense jets and fronts and reveals new eddies with smoother trajectories.
\end{abstract}

\section{Introduction}

The production of accurate sea surface height (SSH) maps is a major challenge for the ocean remote sensing community. Near-real-time and delayed-time multimission reference altimetric $\mathrm{SSH}$ maps are distributed by the AVISO+ (https://www.aviso.altimetry.fr/en/home.html) and the Copernicus Marine Environment Monitoring Service (CMEMS; http://marine.copernicus.eu/) portals and are widely adopted for maritime operation managements, for understanding the ocean surface circulations as well as the response and the monitoring of the ocean surface in a changing climate.

The quality and the robustness of these maps relies on the optimal spatial and temporal sampling of a constellation

Supplemental information related to this paper is available at the Journals Online website: https://doi.org/10.1175/JTECH-D-200030.s1.

Corresponding author: Maxime Ballarotta, mballarotta@ groupcls.com of nadir altimeters as well as the filtering properties of the mapping method. Optimal interpolation (OI) methods have traditionally been chosen to produce these continuous maps in time and space from unevenly spaced altimeter observations (Bretherton et al. 1976; Traon et al. 1998). When the OI mapping is applied to the present-day constellation of nadir altimeters, it allows to retrieve the evolution of mesoscale eddies with typical length scales $\geq 150 \mathrm{~km}$ wavelength and $\geq 30$ days (Ballarotta et al. 2019). Yet, these resolution limits could be reduced by exploiting the high-resolution content of the current along-track altimeter dataset, or with the future mission Surface Ocean and Water Topography (SWOT), which is designed to observe ocean surface topography at a finer scale $(\leq 150 \mathrm{~km})$ than current technologies (Morrow et al. 2019). However, with the choice of orbital parameters set for this future mission, an imbalance between the high spatial resolution and the weaker temporal sampling is expected. As short mesoscale ocean eddies evolve rapidly and nonlinearly, traditional OI methods would smooth out the finest ocean features that could be 
TABLE 1. List of input data and their definition in CMEMS.

\begin{tabular}{|c|c|c|}
\hline Altimeter mission & Name of SEALEVEL CMEMS product & Name of SEALEVEL CMEMS dataset \\
\hline Jason-2 & & $\begin{array}{l}\text { dataset-duacs-rep-global-j2n-phy-13 } \\
\text { dataset-duacs-rep-global-j2g-phy-13 }\end{array}$ \\
\hline$S A R A L$ & & dataset-duacs-rep-global-alg-phy-13 \\
\hline CryoSat-2 & SEALEVEL_GLO_PHY_L3_REP_OBSERVATIONS_008_062 & dataset-duacs-rep-global-c2-phy-13 \\
\hline HaiYang- $2 A$ & & dataset-duacs-rep-global-h2g-phy-13 \\
\hline Jason-3 & & dataset-duacs-rep-global-j3-phy-13 \\
\hline Sentinel-3 & & dataset-duacs-rep-global-s3a-phy-13 \\
\hline
\end{tabular}

observed by the future altimetry mission between revisits of the satellite.

To compensate for the lack of observations and reconstruct the missing signal in time, Ubelmann et al. (2015) proposed the dynamic optimal interpolation (DOI) approach as a technique for a future high-resolution altimetric mapping. The DOI approach was tested on idealized cases (i.e., an idealized SSH field extracted from an ocean model), showing an increased resolution limit of coherent structures toward shorter wavelengths and a reduction of the interpolation error in highly turbulent regions compared to traditional linear optimal interpolation (LOI) mapping (Ubelmann et al. 2015, 2016; Rogé et al. 2017). Based on these observing system simulation experiments (OSSEs), these studies concluded that DOI would allow us to reconstruct the part of meso to submesoscale eddies that are smoothed with the conventional LOI-based mapping approach.

We propose here to further extend the validation of the DOI method by applying it to current along-track altimetry products and investigate its performance in retrieving smaller ocean features that are smoothed by the traditional LOI. To do this, several experimental SSH products based on the DOI method were here designed and evaluated against standard optimal interpolation SSH maps distributed by CMEMS. The paper is organized as follows: Data and methods are presented in section 2. Qualitative and quantitative assessments of the DOI approach are presented in section 3. Finally, the benefits and limitations of the DOI approach are discussed in section 4 .

\section{Experimental design}

a. Data

We produced SSH maps from the merging of unfiltered along-track sea level anomaly products (level 3) for the following altimetry missions: Jason-2, Jason-3, Sentinel-3, Satellite with Argos and Altika (SARAL), CryoSat-2, and HaiYang-2A (see Table 1). These along-track data are distributed by CMEMS (http://marine.copernicus.eu/) and benefit from data homogenization and cross calibration based on standards recommended for altimetry products by different agencies and expert groups such as the Ocean Surface Topography Science Team (OSTST), ESA Quality Working Groups and ESA SL_cci project members. A full description of the processing of these level-3 products is available in the sea level product user manual (http://marine.copernicus.eu/documents/ PUM/CMEMS-SL-PUM-008-032-062.pdf), in the quality information document (http://marine.copernicus.eu/documents/ QUID/CMEMS-SL-QUID-008-032-062.pdf) as well as in Taburet et al. (2019).

\section{b. Methods}

In the present study, two series of SSH maps, constructed with the Jason-2, Jason-3, Sentinel-3, SARAL, and HaiYang- $2 A$ along-track datasets, are generated and compared:

1) $\mathrm{SSH}$ maps produced with similar protocol as for the reference Data Unification and Altimeter Combination System (DUACS) delayed-time 2018 (DT2018) global ocean-multimission altimeter satellite gridded SSHs (Taburet et al. 2019) distributed by CMEMS. Note that the maps tested here are computed specifically for this study, keeping one altimeter mission (here CryoSat-2) out to allow an independent assessment of the mapping performance. These maps are constructed from a LOI method (Bretherton et al. 1976; Traon et al. 1998) based on the a priori statistical knowledge of the SSH fields (e.g., covariance model, correlation length scale, $\mathrm{SSH}$ variance, noise level). The LOI approach used for the DUACS-DT2018 processing is extensively described in section 2.2.6 of Pujol et al. (2016) as well as in Taburet et al. (2019).

2) The experimental regional maps based on the DOI approach. The DOI approach has the advantage of taking nonlinear processes into account. It includes both statistical and physical (dynamical) knowledge of the field to map. It is based on forward/backward transport of the SSH field by a nonlinear quasigeostrophic propagator conserving the potential vorticity. The covariance model is hence dynamically propagated, and the mapping is undertaken with similar system 


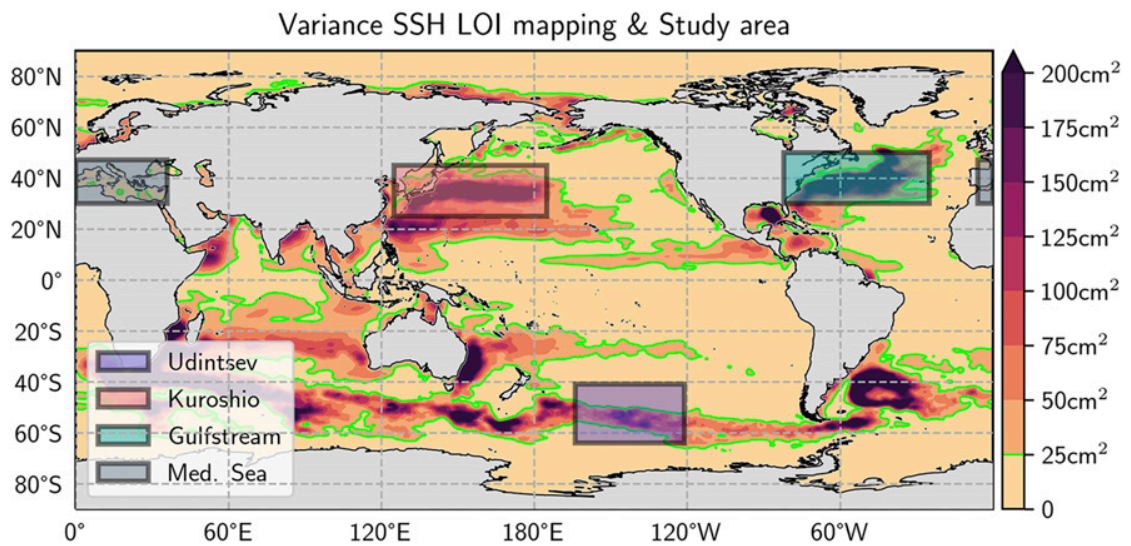

FIG. 1. Map of the mean variance of SSH and spatial extent of the four study areas: the Gulf Stream, the Kuroshio, the Udintsev, and the Mediterranean Sea. The green contour is the $25 \mathrm{~cm}^{2}$ LOI SSH variance contour.

inversion as with classical OI technique. Ubelmann et al. (2015, 2016) and Rogé et al. (2017) described and validated the DOI method by means of OSSE.

\section{c. Study areas: Spatial and temporal coverage}

Figure 1 shows the mean variance of SSH and the spatial coverage of the four regions of interest where the DOI was applied: the Gulf Stream and its extension $\left(30^{\circ}-50^{\circ} \mathrm{N}, 82^{\circ}-25^{\circ} \mathrm{W}\right)$, the Kuroshio region and its extension $\left(25^{\circ}-45^{\circ} \mathrm{N}, 125^{\circ} \mathrm{E}-175^{\circ} \mathrm{W}\right)$, a portion of the Antarctic Circumpolar Current called the Udintsev region $\left(64^{\circ}-41^{\circ} \mathrm{S}\right.$, $\left.164^{\circ}-121^{\circ} \mathrm{W}\right)$ and the Mediterranean Sea $\left(30^{\circ}-47^{\circ} \mathrm{N}\right.$, $\left.6^{\circ} \mathrm{W}-37^{\circ} \mathrm{E}\right)$. The temporal coverage is the period from 1 January 2015 to 31 December 2017. For the quantitative assessment, we focus on the 1-yr period from 1 January to 31 December 2017. In the following section, we focus on the evaluation of the $\mathrm{SSH}$ maps produced for the Gulf Stream system. We refer the reader to the online supplementary material for the assessments of the DOI in the Kuroshio, the Udintsev and the Mediterranean Sea regions. The Gulf Stream region includes various oceanic regimes such as the highly turbulent mean path of the Gulf Stream current and low-energy coastal and offshore regions. We arbitrary distinguished these two regimes by the $25 \mathrm{~cm}^{2}$ variance contour calculated from the reconstructed SSH DT2018 maps (green colored line in Fig. 1).

\section{Results}

The performance of the DOI against the LOI approach is assessed qualitatively and quantitatively using independent data. It should be noted that for the quantitative assessments, we have designed an observing system experiment (OSE) based on the comparison of SSH maps with independent SSH along the track. In this context, specific SSH maps were computed for both the DOI and LOI methods using the Jason-2, Jason-3, Sentinel-3, $S A R A L$, and HaiYang-2A datasets, and one dataset (CryoSat-2) was set aside for independent evaluation.

\section{a. Qualitative assessment}

\section{COMPARISON OF MAPS WITH SEA SURFACE TEMPERATURE}

To illustrate the improved mesoscale structures and front features associated with the dynamic approach, DOI and LOI maps are compared to the observed highresolution sea surface temperature (SST) fields from the CATSAT fisheries remote sensing system (http:// www.catsat.com/ocean-data/). Figure 2 represents an example of the SST on 16 August 2015 with SSH contours superimposed for the LOI and the DOI reconstructions (respectively, Figs. 2a and 2b). This diagnostic illustrates how the SSH maps constructed with the DOI have improved positioning and amplitude of mesoscale and front structures. Many larger structures are similarly reconstructed with the two mapping techniques. The small eddy structures annotated with the numbers 1 and 2 are better resolved with DOI than with LOI. Additionally, the SSH contours follow better the SST front in the upper part of the meander (annotated with number 3) with the DOI than with the LOI approach.

\section{b. Quantitative assessment}

\section{1) COMPARISON OF MAPS WITH INDEPENDENT ALONG-TRACK DATA}

Our first diagnostic evaluates the difference in SSH between the two mapping techniques and the independent along-track products. Figure 3 illustrates the quantitative performance of the DOI method compared to the LOI 

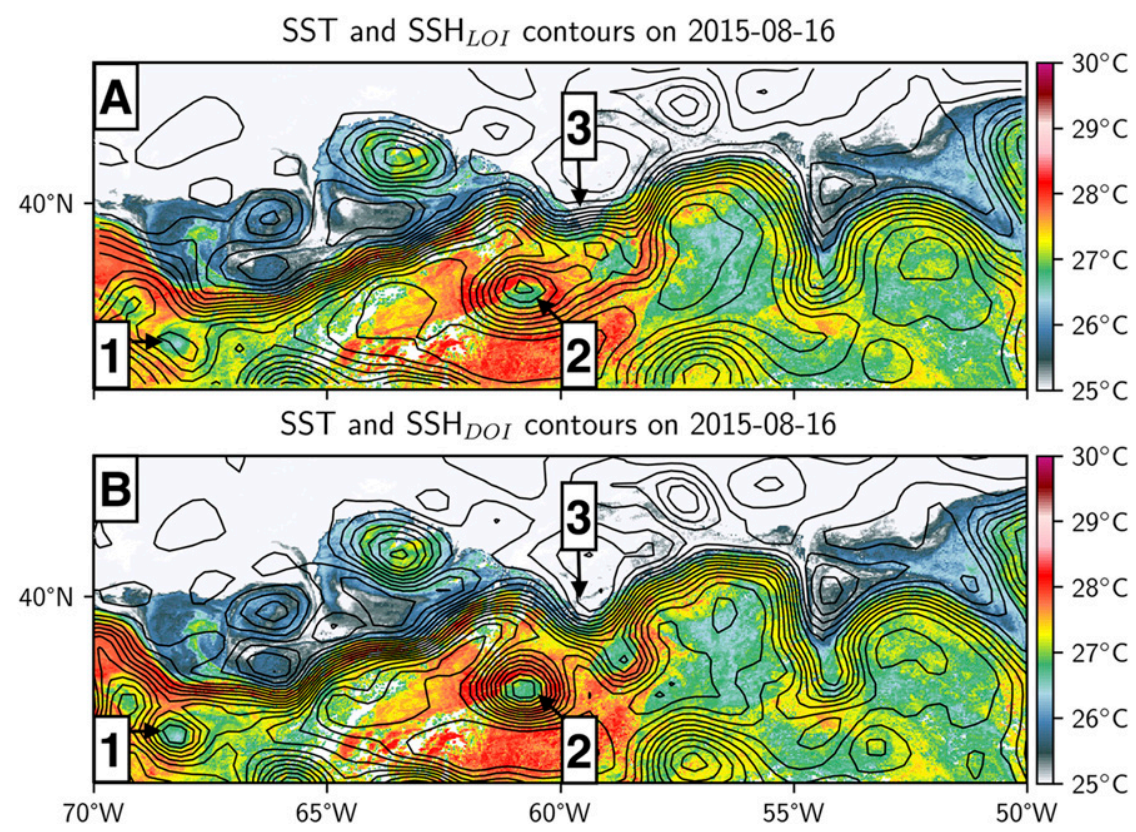

FIG. 2. Maps of sea surface temperature from the CATSAT fisheries remote sensing system superimposed with the SSH contours from (a) the LOI and (b) the DOI reconstructions on 16 Aug 2015.

method. Figure 3 a represents, in $1^{\circ} \times 1^{\circ}$ boxes, the mean gain/loss of variance of the error $\Delta \sigma_{\mathrm{err}}$ between the DOI maps and the independent CryoSat-2 alongtrack data, relatively to the variance of error between the DUACS-DT2018 maps and the same independent CryoSat-2 along-track data. Figure $3 \mathrm{~b}$ shows a similar calculation for the gain/loss in correlation $\Delta R$ between the two mapping techniques. The details of these metrics are provided in the appendix. A negative value of $\Delta \sigma_{\mathrm{err}}$ (in blue) means a reduction of the differences between gridded and along-track SSH when DOI products are considered. A positive value for $\Delta R$ (in red) means a gain in correlation between gridded and along-track SSH when DOI products are considered. We found that the DOI approach reduces mapping errors in highly turbulent regions (for $\mathrm{SSH}$ variance $\geq 25 \mathrm{~cm}^{2}$, green contour). The average reduction of the variance of the error over the Gulf Stream region is $\approx 10 \%$. This quantitative assessment also indicates that the amplitude of the SSH reconstruction with the LOI is better than the DOI method in low variability regions (along the north American coast and near the central North Atlantic basin). Over all regions in the domain of Fig. 3, the correlation is improved by $\approx 5 \%$, suggesting better phasing agreement between the reconstructed SSH and the independent SSH along track. The correlation is deteriorated at isolated points along the continental slope and at the domain boundary, probably related to the boundary condition in the quasigeostrophic model.

\section{2) SPECTRAL ANALYSIS AND EFFECTIVE RESOLUTION}

We performed a spectral analysis between the two mapped products and the independent along-track dataset to estimate the level of performance at different wavelengths. The power spectral densities, the magnitude squared coherences between maps and independent along track and the signal-to-noise ratio (SNR) computed over the Gulf Stream region are shown in Fig. 4. The spectral analysis shows that the DOI SSH signal is in better agreement (both in amplitude and in coherence/phase) with the independent along track for wavelengths $\geq 100 \mathrm{~km}$ than the LOI SSH signal (Figs. 4a,b). This is also demonstrated by the higher SNR with DOI than with LOI (Fig. 4c). The mean effective resolution (computed as the wavelength where SNR $=2$ ) is $\approx 145 \mathrm{~km}$ wavelength with the LOI approach. The mean effective resolution is $\approx 133 \mathrm{~km}$ wavelength with the DOI approach, which corresponds to a mean gain of $\approx 8 \%$.

As in Ballarotta et al. (2019), we estimated the geographical distribution of the effective resolution of the LOI mapping as well as the geographical distribution of the resolution gain/loss with the DOI approach (Fig. 5). The geographical distribution of the effective resolution of the LOI mapping ranges from 100 to $200 \mathrm{~km}$ wavelength in the Gulf Stream region, with short wavelengths found near the U.S. coast and larger resolution found in 

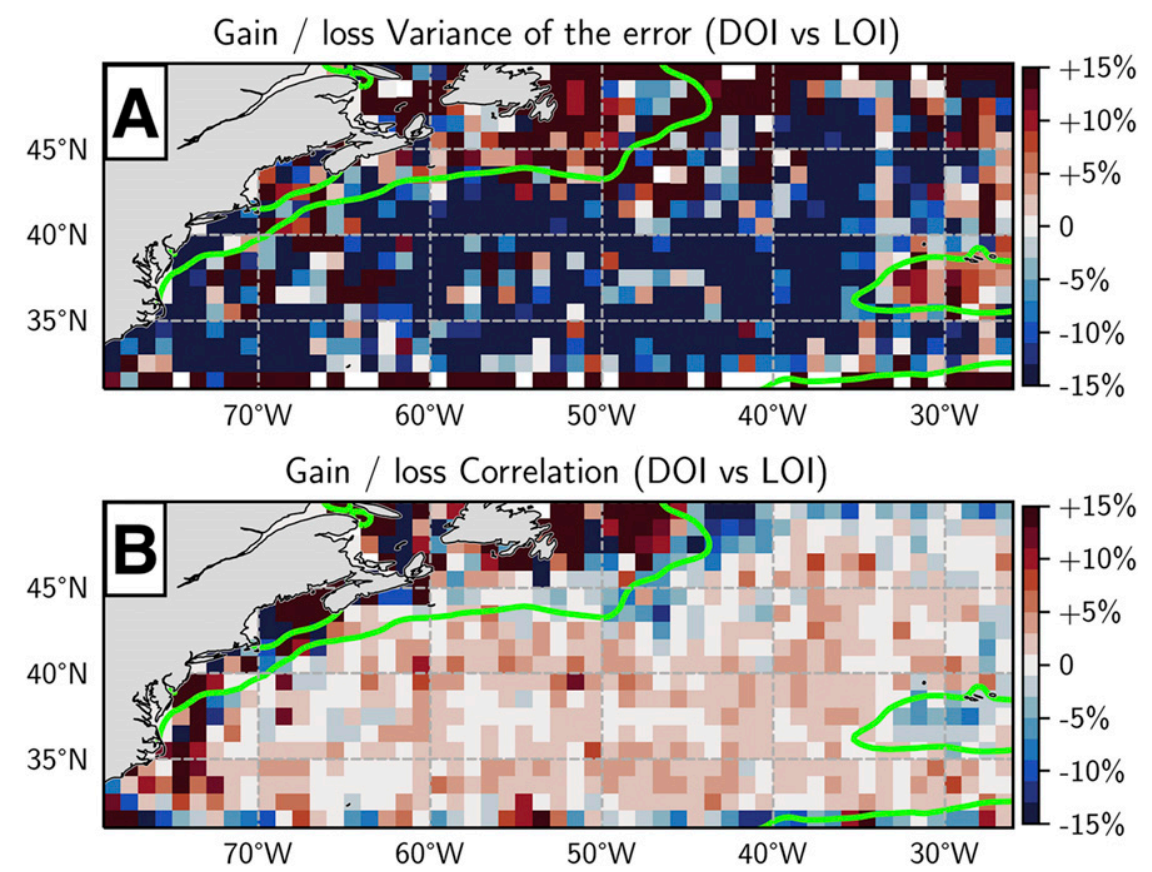

FIG. 3. Maps of (a) gain $(+)$ and loss $(-)$ of variance of the error between DOI and LOI (negative values indicate a reduction of the differences between gridded and along-track SSH when DOI product is considered), and (b) gain $(+)$ and loss $(-)$ of correlation between DOI and LOI (positive values indicate a gain of correlation between gridded and along-track SSH when DOI product is considered). Prior to the statistical analysis, a spatial filtering for wavelength $[65,500] \mathrm{km}$ is done on the data to isolate the mesoscale frequency bands. The green line is the $25 \mathrm{~cm}^{2}$ LOI SSH contour.

the open ocean (Fig. 5a). The resolution gain with the DOI method can be as large as $15 \%$ in the mean path of the Gulf Stream current (Fig. 5b). Resolution loss $(\geq 5 \%)$ is found near the U.S. coast and in the upper part of the domain.

\section{Discussion and conclusions}

The present study continues the analysis presented by Ubelmann et al. (2016) for validating the DOI approach. Their study showed that the DOI method applied to simulated data reduced the mapping error $\left(\mathrm{SSH}_{\text {model }}\right.$ minus $\mathrm{SSH}_{\text {map }}$ ) in highly turbulent regions compared with the traditional LOI mapping method. Here we tested and reported the performance of the DOI approach using realistic altimeter sampling and errors, over a larger study area than previously tested in Ubelmann et al. (2016). Four regional configurations (Gulf Stream, Kuroshio, portion of the Antarctic Circumpolar Current and the Mediterranean Sea) were set up to evaluate these highresolution gridded SSH maps (see supplementary material for evaluation of these other regions). A series of validations and comparisons with independent dataset were carried out to assess the performances of the DOI mapping against the reference CMEMS products. The main outcomes are as follows:

1) We found improvements with DOI in the reconstruction of the mesoscale structures of intense jets, revealing new eddies and better positioning of fronts

2) Quantitative evaluations indicate a $10 \%-15 \%$ reduction in mapping error $\left(\mathrm{SSH}_{\text {independent-along-track }}\right.$ minus $\mathrm{SSH}_{\text {map }}$ )

3) Spectral analysis shows that the DOI mapping can reconstruct features down to $133 \mathrm{~km}$ wavelength in the Gulf Stream region. This represents a gain in resolution of $8 \%$ compared to the LOI mapping

4) However, it is challenging to outperform the CMEMS LOI SSH reference mapping in low-energy and coastal areas

Similar conclusions were drawn for the Kuroshio, the Udintsev, and the Mediterranean Sea regions (see supplemental material). The present study thus demonstrates the possibility of applying the DOI approach with real altimetry observations in various highly turbulent regions of the World Ocean. It also consolidates the findings obtained from OSSEs by Ubelmann et al. (2016) and Rogé et al. (2017). Despite these good performances 

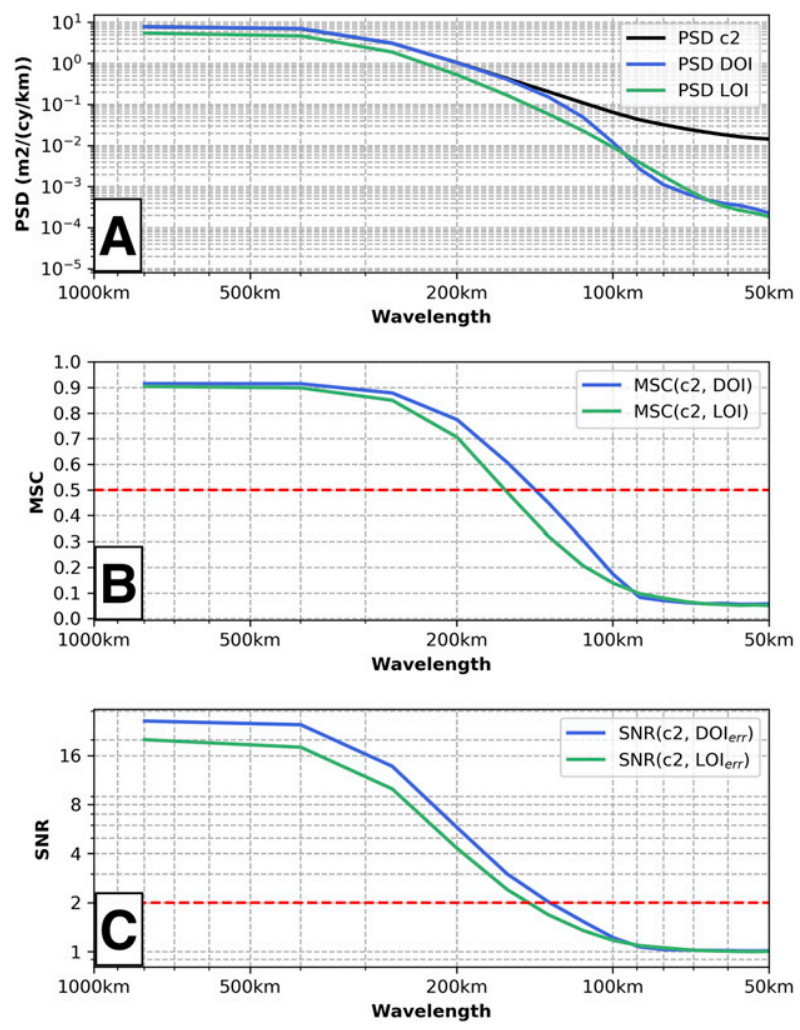

FIG. 4. Spectral analysis over the Gulf Stream region: (a) mean power spectral densities for the independent along-track SSH from CryoSat-2, DOI SSH maps, and LOI SSH maps; (b) magnitude squared coherence between independent along-track SSH and each SSH reconstruction; and (c) signal-to-noise ratio for each mapping methods.

of the DOI approach, several limitations must be discussed. Additional efforts are indeed required to improve the mapping in coastal and low-variability regions. As in Rogé et al. (2017), we found that the DOI method is not yet adapted to coastal areas, where the barotropic mode of the sea surface variability is strong, and close to quasigeostrophic (QG) boundaries. Besides the fact that it is difficult to map the SSH in ocean regions with low variability, the question of the feasibility of applying the QG model in all regions is also open. We already know that QG dynamics are not appropriate in the intertropical region, which can potentially be resolved by other mapping approaches (such as an equatorial waveguide approach).

Nevertheless, we have here demonstrated that increasing the resolution of SSH maps produced from the merging of high-resolution satellite observations requires a smooth transition from the "current" statistical mapping process (objective analysis) to dynamic interpolation of topography. This DOI technique is essentially a hybrid between the current DUACS LOI mapping process and a sophisticated combination of ocean models and data assimilation method [e.g., Mercator Ocean Global Ocean Reanalysis and Simulation (GLORYS) 12v1 reanalysis product], as it exploits the predictability of topography in the ocean. These DOI level-4 SSH products benefit from a better dynamical propagation, while remaining constrained exclusively by satellite topography observations. This method strengthens the interpolation capacity in the time domain, allowing the exploitation of smaller-scale signals in the along track, unlike statistical interpolation, which is forced to smooth the signal because the altimeter coverage is insufficient.

We have applied unfiltered along-track altimetry data here, which include finescale SSH signals that could be propagated by the QG dynamic but also errors. Different SSH error sources will affect the mapping from along-track altimetric data, which are not present in previous OSSE DOI versus LOI evaluations. For example, nonrepeating altimetric missions have their SSH calculated with respect to a gridded mean sea surface (MSS), and over sharp bathymetric gradients this could introduce increased SSH errors at spectral wavelengths of $30-80 \mathrm{~km}$ (Pujol et al. 2018). Locally, these small MSS-induced errors in the SSH are smoothed out in the LOI analysis, but could be propagated by the QG model in the DOI. This may contribute to reducing the DOI performance in regions of sharp bathymetric gradients, for example, close to coasts. We expect an improved global MSS estimate after 1-3 years of the future SWOT mission, but these MSS errors remain a problem with our current nonrepeat altimetry missions within the constellation. Similarly, internal tides can have similar space scales to the mesoscale structures in altimetric data, and again the LOI will smooth these anomalies and the DOI will propagate them via QG dynamics. Note that we have here focused on unfiltered data in order to include the small-scale transient eddies that could be propagated by the QG dynamic. Filtered along-track data could also be considered in the DOI mapping, resulting in a smoothing/ filtering of these small-scale transient eddies.

Finally, it should be mentioned that the DOI approach is also not the unique method that can help in retrieving smaller-scale oceanic features or the missing signals in the state-of-the-art SSH mapping system such as the DUACS system. By combining various type of sensors (altimeter data and in situ drifter measurements), Mulet et al. (2020) demonstrated that multivariate objective analysis can help to refine the mapping of the SSH in the Gulf of Mexico (with resolution gain of $10 \%$ ). The multiscale mapping approach developed in the framework of the Sea Surface Kinematics Multiscale Monitoring (SKIM) mission, simple data assimilation methods (e.g., backward-forward nudging; Guillou et al. 2020) or machine learning approaches (Lguensat et al. 2017; Fablet et al. 2017) may also offer the possibility to map 

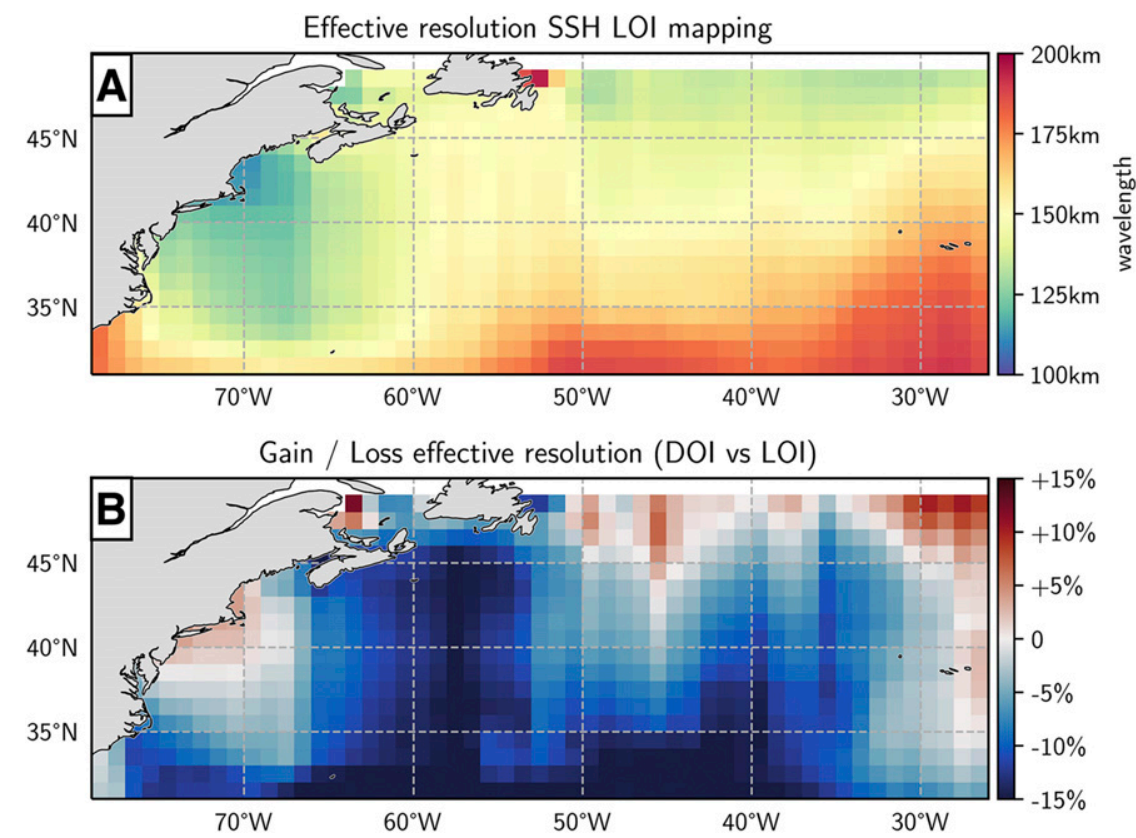

FIG. 5. Maps of (a) effective resolution of the LOI SSH reconstructions and (b) gain (+) and loss $(-)$ of effective resolution between DOI and LOI (negative values mean finer resolution with DOI than with LOI).

the various and complex regimes that exist in the real motion of the ocean surface.

Acknowledgments. This study was funded by a CNES R\&D contract. The authors would like to thank two anonymous referees for their valuable comments and suggestions.

Data availability statement. The unfiltered along-track sea level anomaly products (level 3) used in this study are freely available on the CMEMS portal (http:// marine.copernicus.eu/). The DOI maps produced in these study will be made available on the AVISO+ website.

\section{APPENDIX}

\section{Quantitative Assessment: Methods and Metrics}

a. Statistical approach: Comparison between maps and independent along-track data

This diagnostic follows three main steps:

1) Gridded $\mathrm{SSH}$ data $\left(\mathrm{SSH}_{\text {reconstruction }}\right)$ are interpolated to the locations of the independent SSH along-track data $\left(\mathrm{SSH}_{\text {true }}\right)$, georeferenced by their longitude, latitude and time. Note that the along-track SSHs are constructed using a procedure similar to level-3 CMEMS products.
2) The difference $\Delta \mathrm{SSH}=\mathrm{SSH}_{\text {reconstruction }}-\mathrm{SSH}_{\text {true }}$ (representative of the mapping errors) is computed.

3) A statistical analysis on the $\Delta \mathrm{SSH}$ and $\mathrm{SSH}$ signals is performed in $1^{\circ}$ longitude $\times 1^{\circ}$ latitude boxes. Prior to the statistical analysis, a filtering operation can be applied to isolate frequency bands of interest. For example, one can perform the analysis on the spatial range $[65,500] \mathrm{km}$ typically representative of the mesoscale oceanic signal.

In each grid box, the expressions of the variance of the error $\sigma_{\text {err }}^{2}$ and correlation $R$ between $\mathrm{SSH}_{\text {true }}$ and $\mathrm{SSH}_{\text {reconstruction }}$ are computed. The mathematical formulations are

$$
\begin{aligned}
\sigma_{\text {err }}^{2}(i) & =\frac{\sum_{i=1}^{N}[\Delta \mathrm{SSH}(i)-\overline{\Delta \mathrm{SSH}(i)}]^{2}}{N}, \\
R(i) & =\frac{\operatorname{Cov}\left(\mathrm{SSH}_{\text {true }}, \mathrm{SSH}_{\text {reconstruction }}\right)}{\sigma\left(\mathrm{SSH}_{\text {true }}\right) \sigma\left(\mathrm{SSH}_{\text {reconstruction }}\right)},
\end{aligned}
$$

where $\mathrm{SSH}_{\text {true }}$ is the reference SSH field (independent

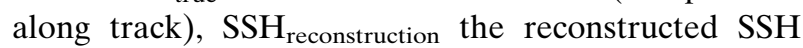
field (SSH maps), $i$ denotes the spatiotemporal index, $N$ the total number of observation, Cov is the covariance function, and $\sigma$ is the standard deviation. 
The quantitative assessment of the performance of the DOI versus LOI mapping is then given by 1) the gain/loss of variance of the error:

$$
\frac{\sigma_{\mathrm{err}_{\mathrm{DOI}}}^{2}-\sigma_{\mathrm{err}_{\mathrm{LOI}}}^{2}}{\sigma_{\mathrm{err}_{\mathrm{LOI}}}^{2}} \times 100 .
$$

It is expressed as a percentage (\%). A negative value means an improvement (i.e., the DOI product is closer to the along-track product than the LOI product), a positive value means a deterioration (i.e., the LOI product is closer to the along-track product than the DOI product).

And 2) by the gain/loss of correlation:

$$
\frac{R_{\text {DOI }}-R_{\text {LOI }}}{R_{\text {LOI }}} \times 100
$$

It is expressed in percentage (\%). A positive value means an improvement (i.e., the DOI product is closer to the along-track product than the LOI product), a negative value means a deterioration (i.e., the LOI product is closer to the along-track product than the DOI product).

\section{b. Spectral approach: Comparison between maps and independent along-track data}

A spectral analysis is applied to altimetry dataset and is described, for example, in Ballarotta et al. (2019). We recall here the main processing steps:

Mapped SSH data $\left(\mathrm{SSH}_{\text {reconstruction}}\right)$ are interpolated to the locations of the independent along-track $\mathrm{SSH}$ data $\left(\mathrm{SSH}_{\text {true }}\right)$. Along-track and interpolated data are divided into $800-\mathrm{km}$-long segments overlapping every $200 \mathrm{~km}$. Each segment is saved in a database and referenced by its median (longitude, latitude) coordinates Finally, between latitudes $90^{\circ} \mathrm{N}$ and $90^{\circ} \mathrm{S}$ and between longitudes $0^{\circ}$ and $360^{\circ} \mathrm{E}$, we consider $10^{\circ}$ longitude $\times$ $10^{\circ}$ latitude averaging boxes $\left(5^{\circ}\right.$ longitude $\times 5^{\circ}$ latitude averaging boxes for the Mediterranean Sea) every $1^{\circ}$ incremental step. All available segments referenced within the $10^{\circ} \times 10^{\circ}$ box are selected to compute the power spectral densities, cross spectral density, magnitude squared coherence, based on the Welch (1967) method. Prior to spectral computation, signals are detrended and we applied a Hanning window. The signalto-noise ratio is then deduced from the power spectral density of the along-track $\operatorname{PSD}\left(\mathrm{SSH}_{\text {true }}\right)$ and the power spectral density of the error $\operatorname{PSD}(\Delta \mathrm{SSH})$.

In the spectral domain, the main metrics consist of the power spectral density (PSD), the magnitude squared coherence (MSC), SNR and the spectral ratio (SR). The mathematical expression of the MSC, and SNR are formulated below. For the mathematical formulation of the PSD, see Welch (1967):

$$
\begin{aligned}
\operatorname{MSC}(\lambda) & =\frac{\left|\mathrm{CS}\left(\mathrm{SSH}_{\text {reconstruction }}, \operatorname{SSH}_{\text {true }}\right)(\lambda)\right|^{2}}{\operatorname{PSD}\left(\operatorname{SSH}_{\text {reconstruction }}\right)(\lambda) \operatorname{PSD}\left(\mathrm{SSH}_{\text {true }}\right)(\lambda)}, \\
\operatorname{SNR}(\lambda) & =\frac{\operatorname{PSD}(\Delta \mathrm{SSH})(\lambda)}{\operatorname{PSD}\left(\mathrm{SSH}_{\text {true }}\right)(\lambda)},
\end{aligned}
$$

where CS denote the cross-spectral analysis operator, PSD is the power spectral density, and $\lambda$ is the wavelength.

\section{REFERENCES}

Ballarotta, M., and Coauthors, 2019: On the resolutions of ocean altimetry maps. Ocean Sci., 15, 1091-1109, https://doi.org/ 10.5194/os-15-1091-2019.

Bretherton, F., R. Davis, and C. Fandry, 1976: A technique for objective analysis and design of oceanographic instruments applied to MODE-73. Deep-Sea Res., 23, 559-582, https:// doi.org/10.1016/0011-7471(76)90001-2.

Fablet, R., P. H. Viet, and R. Lguensat, 2017: Data-driven models for the spatio-temporal interpolation of satellite-derived SST fields. IEEE Trans. Comput. Imaging, 3, 647-657, https:// doi.org/10.1109/TCI.2017.2749184.

Guillou, F. L., and Coauthors, 2020: Mapping altimetry in the forthcoming swot era by back-and-forth nudging the quasigeostrophic dynamics. EGU General Assembly, European Geosciences Union, 19273, https://doi.org/10.5194/egusphereegu2020-19273.

Lguensat, R., P. Tandeo, P. Ailliot, M. Pulido, and R. Fablet, 2017: The analog data assimilation. Mon. Wea. Rev., 145, 4093-4107, https://doi.org/10.1175/MWR-D-16-0441.1.

Morrow, R., L.-L. Fu, F. D'Ovidio, and J. T. Farrar, 2019: Scientists invited to collaborate in satellite mission's debut. Eos, Trans. Amer. Geophys. Union, 100, https://doi.org/10.1029/ 2019EO110423.

Mulet, S., H. Etienne, M. Ballarotta, Y. Faugere, M. Rio, G. Dibarboure, and N. Picot, 2020: Synergy between surface drifters and altimetry to increase the accuracy of sea level anomaly and geostrophic current maps in the Gulf of Mexico. Adv. Space Res., https://doi.org/10.1016/J.ASR.2019.12.024, in press.

Pujol, M., Y. Faugère, G. Taburet, S. Dupuy, C. Pelloquin, M. Ablain, and N. Picot, 2016: DUACS DT2014: The new multi-mission altimeter data set reprocessed over 20 years. Ocean Sci., 12, 1067 1090, https://doi.org/10.5194/os-12-1067-2016.

_ , P. Schaeffer, Y. Faugère, M. Raynal, G. Dibarboure, and N. Picot, 2018: Gauging the improvement of recent mean sea surface models: A new approach for identifying and quantifying their errors. J. Geophys. Res. Oceans, 123, 5889-5911, https://doi.org/10.1029/2017JC013503.

Rogé, M., R. Morrow, C. Ubelmann, and G. Dibarboure, 2017: Using a dynamical advection to reconstruct a part of the SSH evolution in the context of SWOT, application to the Mediterranean Sea. Ocean Dyn., 67, 1047-1066, https://doi.org/ 10.1007/s10236-017-1073-0.

Taburet, G., A. Sanchez-Roman, M. Ballarotta, M. Pujol, J. Legeais, F. Fournier, Y. Faugere, and G. Dibarboure, 2019: DUACS DT2018: 25 years of reprocessed sea level altimetry products. 
Ocean Sci., 15, 1207-1224, https://doi.org/10.5194/os-15-12072019.

Traon, P. L., F. Nadal, and N. Ducet, 1998: An improved mapping method of multisatellite altimeter data. J. Atmos. Oceanic Technol., 15, 522-534, https://doi.org/10.1175/1520-0426(1998) $015<0522$ :AIMMOM $>2.0 . \mathrm{CO} ; 2$.

Ubelmann, C., P. Klein, and L. Fu, 2015: Dynamic interpolation of sea surface height and potential applications for future highresolution altimetry mapping. J. Atmos. Oceanic Technol., 32, 177-184, https://doi.org/10.1175/JTECH-D-14-00152.1.
B. Cornuelle, and L. Fu, 2016: Dynamic mapping of along-track ocean altimetry: Method and performance from observing system simulation experiments. J. Atmos. Oceanic Technol., 33, 1691-1699, https://doi.org/10.1175/ JTECH-D-15-0163.1.

Welch, P., 1967: The use of the fast Fourier transform for the estimation of power spectra: A method based on time averaging over short, modified periodograms. IEEE Trans. Audio Electroacoust., 15, 70-73, https://doi.org/10.1109/ TAU.1967.1161901. 